\title{
Tanque Hidráulico Experimental para cálculo de perda de carga em tubulações
}

Renato Letizia Garcia ${ }^{1}$, Daiane Acosta Falcão ${ }^{1}$, José André Della Giustina Neto ${ }^{2}$, Cristiane Cassales Pibernat ${ }^{1}$, Jorge Rodolfo Zabadal $^{3}$

Universidade Estadual do Rio Grande do Sul, Unidade de Novo Hamburgo, Rua Inconfidentes, 395, Bairro Primavera, Novo Hamburgo, RS, Brasil. E-mails: renato-garcia@uergs.edu.br, daia_falcao@hotmail.com, cristiane-pibernat@uergs.edu.br Universidade Federal do Rio Grande do Sul, Centro de Biotecnologia, Departamento de Biologia Molecular e Biotecnologia do Instituto de Biociências, Av. Bento Gonçalves, 9500 - Prédio 43.431 - Lab. 212, Campus do Vale, CEP 91.501-970 - Porto Alegre - RS - Brasil. E-mail: jgn@tecdus.com.br

Resumo - $\mathrm{O}$ artigo descreve a construção de um tanque hidráulico para o cálculo da perda de carga em dutos cilíndricos retos e a execução dos ensaios. $\mathrm{O}$ tanque possui estrutura simples, baixo custo, robustez e versatilidade necessárias para a realização de ensaios de fácil execução, baseados no registro dos tempos associados à variação da altura da coluna de água num tubo vertical. A utilizaçấo deste equipamento em aulas experimentais mostrou-se muito eficaz, possibilitando a comprovação de resultados teóricos a partir da utilização de modelos reais. Os resultados obtidos são apresentados em planilhas eletrônicas, cuja elaboração baseou-se na aplicação dos princípios de conservação de massa e energia no escoamento de fluidos viscosos, e nas equaçóes de perda de carga distribuída e localizada. O experimento proposto constitui uma ferramenta pedagógica interessante, tendo em vista que sua execução requer a participação ativa dos alunos na realização dos ensaios e na execução de cálculos. A rotina de cálculos, visando comparar valores experimentais perda de carga obtidos experimentalmente com valores teóricos, abrange uma série de conceitos e equações da Mecânica dos Fluidos, propiciando que a aprendizagem desse conteúdo seja feita a partir de um problema real.

Palavras-chave - Perda de carga. Tanque hidráulico. Mecânica dos fluidos.

Abstract - The article describes the construction of a hydraulic tank designed for head loss calculation in straight cylindrical pipeline and the essays execution.
The tank has low cost, simple structure, robustness and versatility required for carrying out easy execution tests, upon the recording of the time associated with changes in height of the water column in a vertical tube. The use of this equipment in practical lessons was very effective, engaging students in carrying out the tests and performing calculations. The obtained results were satisfactory and presented on spreadsheets, whose development consists on applying the principles of the mass and energy conservation in the flow of viscous fluids, as well as equations for continuous and local head losses. This experimental activity is an interesting pedagogical tool, since its implementation requires the active participation of students in carrying out tests calculations. The routine of calculations, aiming to compare head losses experimentally obtained with theoretical values, covers many concepts and equations of Fluid Mechanics, providing an opportunity for learning this subject upon the analysis of a real problem. Keywords - Head loss. Hydraulic tank. Fluid Mechanics.

Recebido em: 11 de março de 2016

Aprovado em: 30 de março de 2016

\section{INTRODUÇÁO}

As disciplinas de Fenômenos de Transporte, que tratam do estudo da transferência de quantidade de movimento, energia e massa, integram o currículo dos cursos de Engenharia, abordando os fundamen- 
tos teóricos de processos mecânicos e industriais como o bombeamento de fluidos, a troca térmica em equipamentos e a separação e purificaçáo de produtos. Tal abordagem é realizada de forma dialogada e expositiva, acrescida da resolução de problemas.

Em alguns cursos, disciplinas experimentais de Fenômenos de Transporte também estão presentes no currículo, propiciando ao aluno uma excelente oportunidade de aprendizado e uma melhor compreensão dos assuntos abordados nas aulas teóricas (D'ANGELO; ZEMP, 2014). Contudo, os experimentos realizados nessas disciplinas, em geral, utilizam equipamentos sofisticados e caros, o que torna essas aulas práticas inacessíveis para muitas instituiçôes de ensino (LUDKE et al., 2013).

No intuito de contornar as dificuldades mencionadas anteriormente, foi elaborada uma aula prática sobre perda de carga no escoamento de fluidos, na disciplina de Laboratórios de Engenharia de Bioprocessos I, do curso de Engenharia de Bioprocessos e Biotecnologia da UERGS em Novo Hamburgo. O experimento proposto foi concebido a partir de um equipamento de baixo custo e de um ensaio de fácil execução, a fim que fosse implementado de forma mais célere no conteúdo programático da disciplina.

\section{FUNDAMENTOS TEÓRICOS}

O balanço de quantidade de movimento para o escoamento de um fluido ideal ou invíscido, cuja viscosidade é nula e, consequentemente, escoa sem dissipação de energia por atrito, é expresso pela equação de Euler (FOX; McDONALD; PRITCHARD, 2004), cuja forma vetorial é:

$$
\rho \cdot \frac{D \vec{V}}{D t}+\nabla p+\rho \cdot \vec{g}=0
$$

Nessa equação, a massa específica, a aceleração da gravidade, a pressáo e a velocidade correspondem, respectivamente, ae.Essa equação diferencial pode ser aplicada sobre uma linha de corrente, pois ela representa a trajetória das partículas de um fluido invíscido (SHAMES, 1996). Na condição de regime permanente, para um fluido incompressível, o resultado dessa integração fornece:

$$
\frac{p}{\rho \cdot g}+\frac{V^{2}}{2 \cdot g}+h=c t e .
$$

A equação (2) retrata a conservação da energia mecânica no escoamento em regime permanente e in- compressível de um fluido ideal, ao longo de uma linha de corrente, sobre o qual não há a realização ou introdução de trabalho externo. Tais restriçôes impedem sua aplicação em problemas de engenharia, pois todo fluido real possui viscosidade. Contudo, a introdução de um termo $\left(\boldsymbol{b}_{\boldsymbol{p}}\right)$ associado à perda de energia produzida pela fricção das partículas do fluido entre si e com as paredes internas que delimitam o escoamento, permite sua utilização em situaçôes reais, na forma:

$$
\frac{p_{1}}{\rho \cdot g}+\frac{V_{1}^{2}}{2 \cdot g}+h_{1}=\frac{p_{2}}{\rho \cdot g}+\frac{V_{2}^{2}}{2 \cdot g}+h_{2}+h_{p}
$$

na qual os índices 1 e 2 , nos termos de velocidade, altura (h) e pressão, estão vinculados, respectivamente, às parcelas de energia cinética, potencial e de pressão em duas seçôes transversais ao escoamento do fluido.

Cabe aqui destacar que, no experimento proposto, as seçôes de entrada e saída do fluido estão abertas para o ambiente, as pressões $\mathrm{p}_{1}$ e $\mathrm{p}_{2}$ são idênticas $\mathrm{e}$ iguais à pressão atmosférica.

Além disso, a velocidade $\mathrm{V}_{1}$ é cerca de $0,3 \%$ de $\mathrm{V}_{2}$ e, consequentemente, a energia cinética na seção de entrada é desprezível, sendo possível rearranjar a equação (3) na forma:

$$
h_{1}-h_{2}=\frac{V_{2}^{2}}{2 . g}+h_{p}
$$

A perda de carga, que corresponde à perda de energia por fricção no escoamento de um fluido viscoso, entre essas duas seçôes, representada pelo termo $\mathbf{h}_{\mathbf{p}}$, está convertida em altura de coluna de fluido e possui dimensão de comprimento linear, assim como os demais termos que integram a equação (4).

A perda de carga em um trecho reto de tubulaçáo é caracterizada como uma perda distribuída, pois seu valor é diretamente proporcional ao comprimento dessa tubulação. As alterações na direção ou no módulo da velocidade do fluido, produzidas por mudanças na área da seção transversal do escoamento ou pela presença de acessórios como válvulas e conexôes, constituem perdas localizadas de um sistema de tubulaçôes. As perdas de carga distribuídas e localizadas podem ser calculadas, respectivamente, pelas seguintes expressões:

$$
\begin{aligned}
& h_{p d}=f \cdot\left(\frac{L}{D}\right) \cdot \frac{V^{2}}{2 \cdot g} \\
& h_{p l}=K \cdot \frac{V^{2}}{2 \cdot g}
\end{aligned}
$$


nas quais $\boldsymbol{f}$ e $\boldsymbol{K}$ são coeficientes adimensionais e $L / D$ é o quociente entre o comprimento e o diâmetro de um tubo reto. Evidencia-se a similaridade entre (5) e (6) pela presença, em ambas, do termo $V^{2} / \mathbf{2}$.g, que caracteriza a proporcionalidade entre a perda de carga e a energia cinética do fluido no escoamento (WHITE, 2006; SCHLICHTING, 2001).

O coeficiente de atrito de Darcy, expresso como $f$ na equação (5), pode ser estimado pelo emprego de equaçōes empíricas ou tabelas que relacionam esse coeficiente com o diâmetro e a rugosidade do tubo e o valor de número de Reynolds $(\boldsymbol{R} \boldsymbol{e})$ do escoamento analisado. Cabe aqui ressaltar que $\boldsymbol{R} \boldsymbol{e}$ é um valor adimensional e corresponde ao quociente entre as forças inerciais e as forças viscosas que atuam no escoamento; e, para escoamentos viscosos em condutos forçados, é fornecido pela expressão a seguir:

$$
R e=\frac{D \cdot V \cdot \rho}{\mu}
$$

$\mathrm{Na}$ equação (7), D é o diâmetro interno do tubo, $\mathrm{V}$ é a velocidade média do escoamento no interior do tubo, e $\nabla$ e $\mu$ são, respectivamente a massa específica e a viscosidade do fluido.

Os valores de $\boldsymbol{K}$ para alguns acessórios são apresentados em tabelas, algumas das quais fornecidas pelos próprios fabricantes. Os dois coeficientes $(f \mathrm{e}$ $K)$ podem ser estimados por meio de ensaios de laboratório.

\section{MATERIAIS E MÉTODOS}

\subsection{Aparato Experimental}

O projeto para construção do tanque iniciou com a pesquisa bibliográfica em sítios na Internet e a realização de consultas a professores e pesquisadores envolvidos no ensino de Mecânica dos Fluidos. Sua execução foi feita em dois módulos: base (estrutura) de aço e tanque propriamente dito.

A Figura 1 é uma representação plana, correspondente a um corte longitudinal, sem escala, do tanque; e nela são indicadas as peças integrantes desse equipamento. A seguir será detalhado o processo de montagem do tanque e, a fim de facilitar sua compreensão, as peças citadas serão referenciadas conforme a numeração presente nessa figura.

O tanque propriamente dito é constituído por um tubo de acrílico transparente (peça 1), com 15 $\mathrm{cm}$ de diâmetro e $1 \mathrm{~m}$ de comprimento, encaixado em um tampão de PVC (peça 3), do tipo utilizado em tubulação de esgoto, ao qual estáo acoplados um flange (peça 4), uma válvula esfera (peça 5), cujo diâmetro é de $3 / 4$ de polegada, e uma conexáo reta (peça 6). A necessidade de uma clara visualização do nível de água resultou no emprego de acrílico transparente no corpo do tanque, em cuja superfície externa foi fixada uma fita métrica.

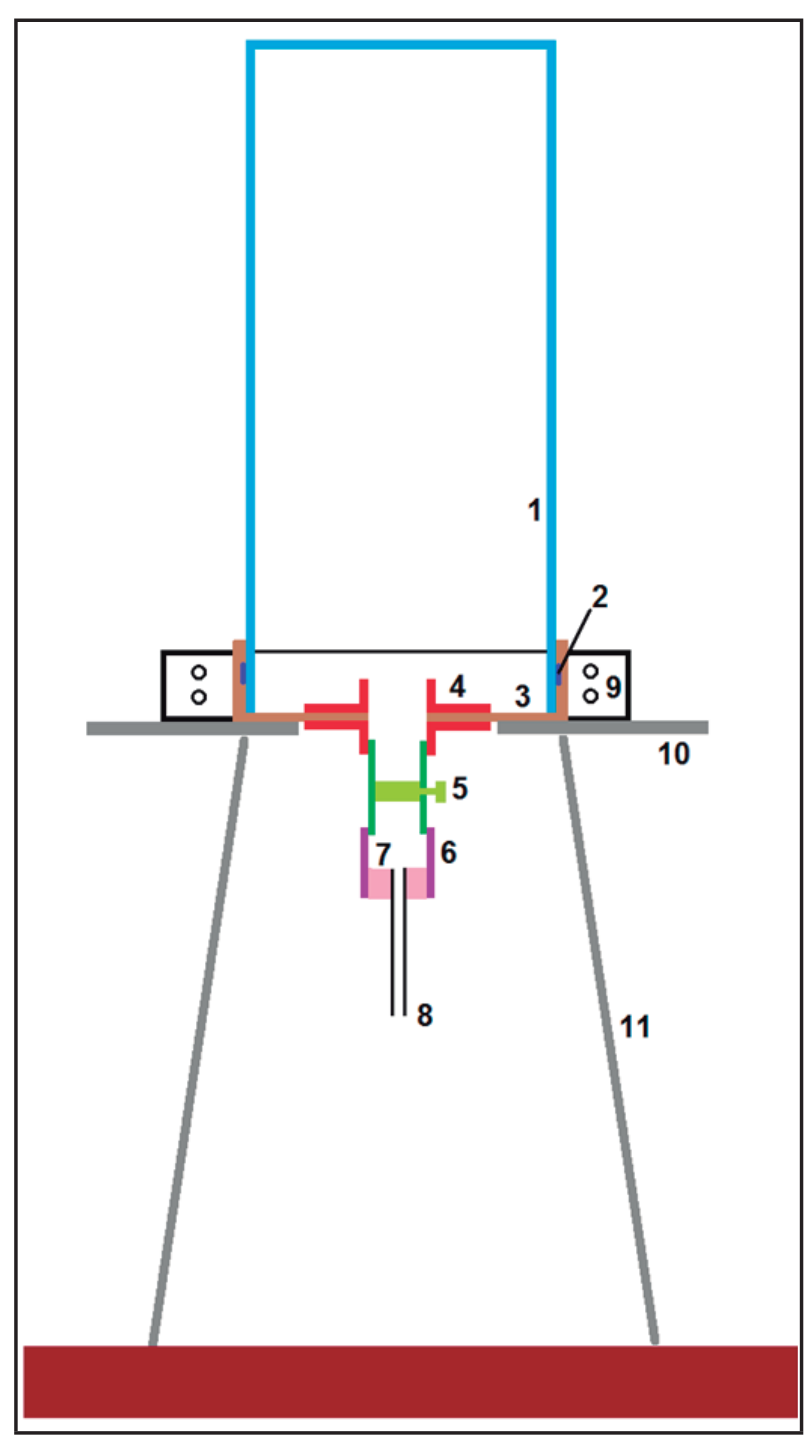

Figura 1 - Corte longitudinal do equipamento utilizado para a realização dos ensaios, detalhando suas peças: (1) tanque de acrílico, (2) anel de borracha de vedação, (3) tampão de PVC, (4) flange de PVC, (5) válvula esfera, (6) conexão reta, (7) tampão rosqueado de PVC, (8) tubo metálico, (9) abraçadeira, (10) base (suporte do tanque), (11) tripé.

A construção da base foi realizada em uma serralheria, sendo que nessa etapa alguns detalhes construtivos foram observados. A inserção de parafusos na extremidade das hastes de sustentação da base (peça 11) permite o nivelamento da estrutura. A utilizaçáo de uma abraçadeira metálica (peça 9), soldada na placa triangular (peça 10) que suporta o tanque, foi 
concebida de modo a regular o aperto do tanque na estrutura metálica, possibilitando uma fixação adequada para a realização dos ensaios e facilitando o desacoplamento e transporte desses módulos.

Uma visão geral do tanque é apresentada na Figura 2; sendo que sua fixação na estrutura metálica e os acessórios acoplados na seçáo de saída do tanque são visualizados nas Figuras 3 e 4, respectivamente.

A fim de reduzir os custos desse equipamento, o flange, a válvula e a conexão reta utilizadas são de PVC. O tubo de acrílico foi desbastado numa das extremidades, de modo a viabilizar seu encaixe no tampão. Utilizou-se um anel de borracha (peça 2) entre essas peças para garantir a vedação. A Figura 5 detalha o tampão que serve de base para o tanque e a inserção do flange nessa peça.

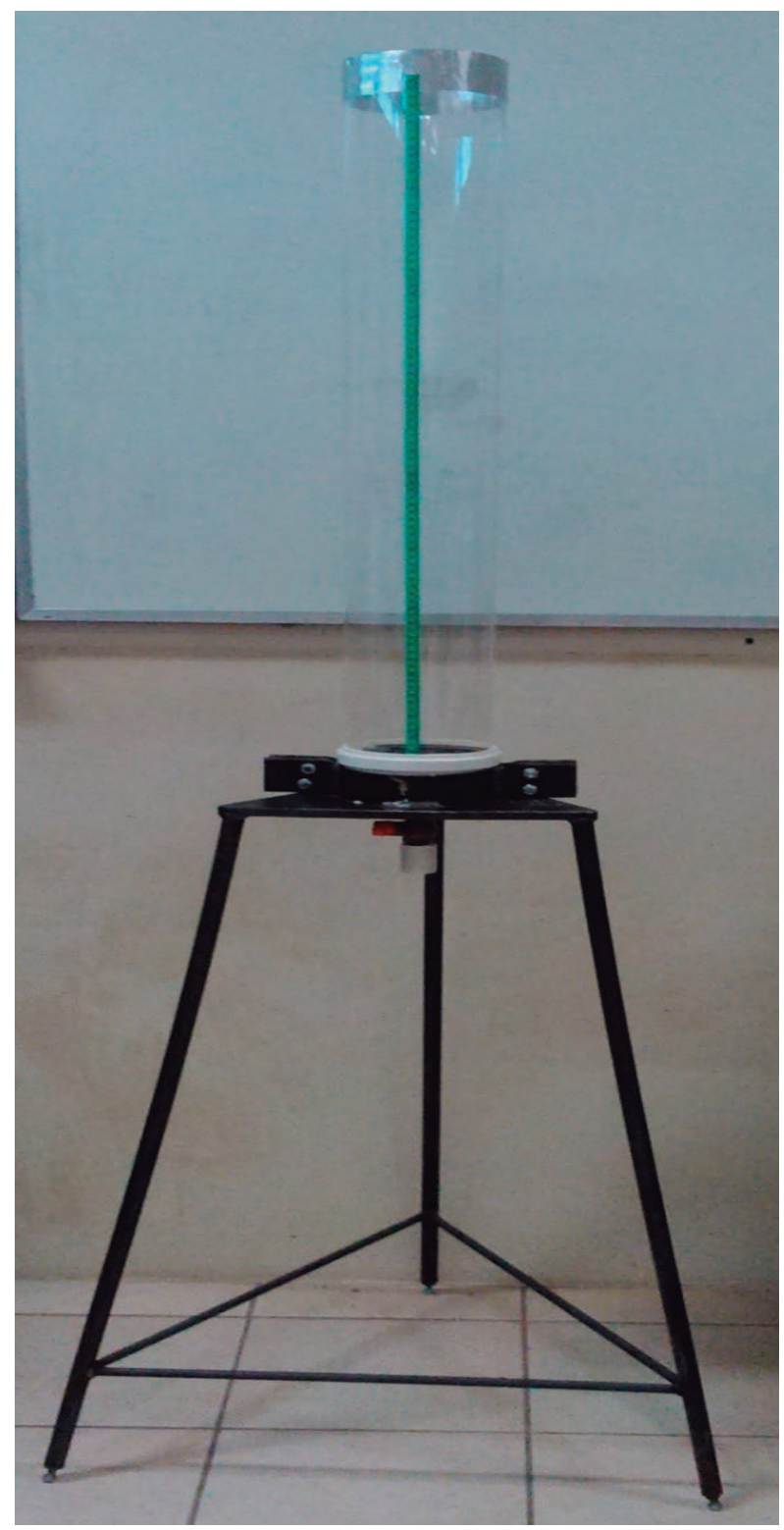

Figura 2 - Visão geral do tanque

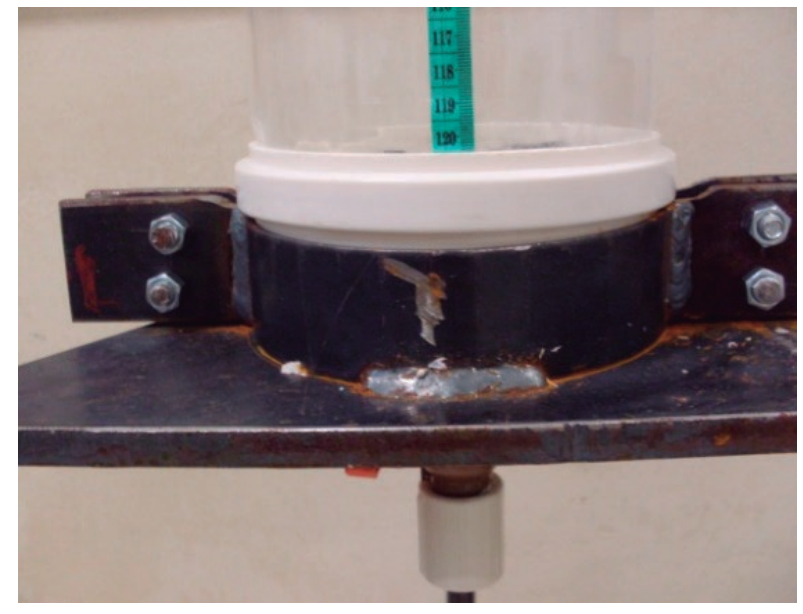

Figura 3 - Abraçadeira de fixaçáo do tanque

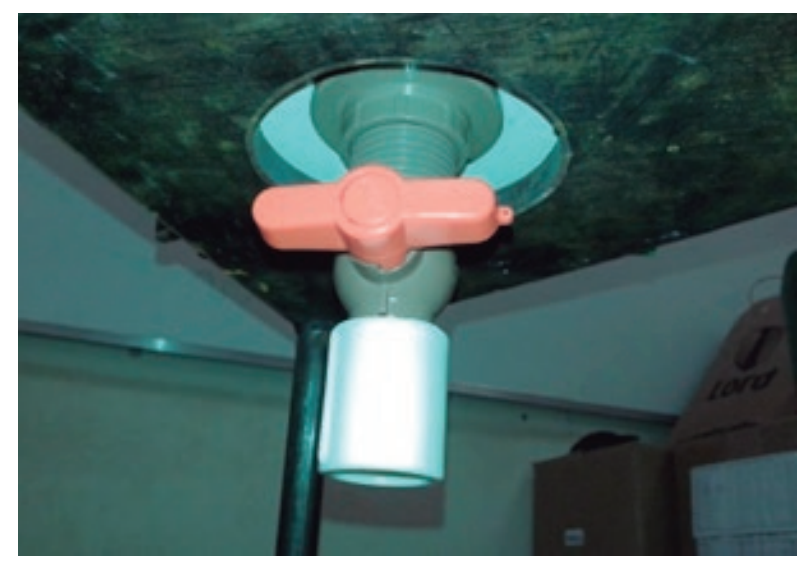

Figura 4 - Vista do fundo do tanque

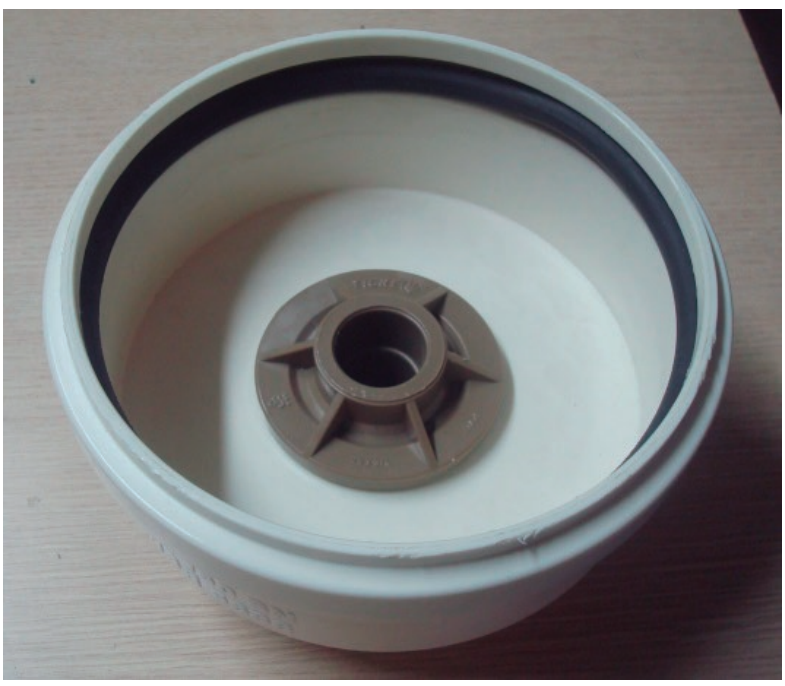

Figura 5 - Detalhe da inserção do flange no tampão

Os tubos (peça 8) empregados no experimento são de aço comercial, com diâmetro interno de $7,2 \mathrm{~mm}$ e comprimentos de 600, 300, 150 e $75 \mathrm{~mm}$. O acoplamento dos tubos no tanque é feito com uma conexão reta, com rosca interna. Uma das extremidades de cada tubo foi inserida num tampão rosqueado de PVC 
(peça 7), e o espaço anular entre as peças foi preenchido com cola epóxi, obtendo-se assim um conjunto de tubulaçôes intercambiáveis, ilustradas na Figura 6.

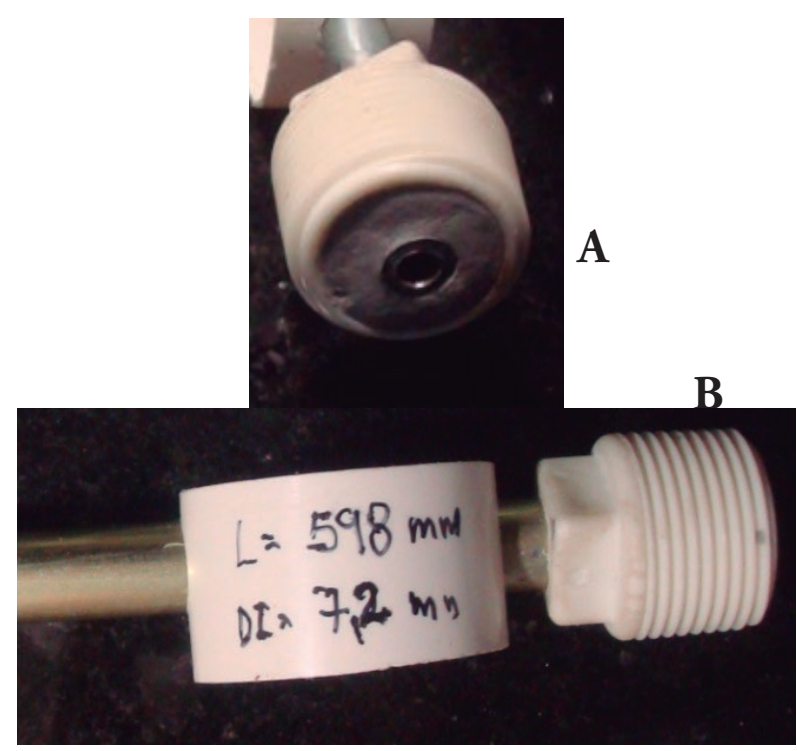

Figura 6 - Fotografia dos tubos utilizados: (a) preenchimento do espaço anular entre o tubo e o tampáo; (b) rosca externa do tampão que possibilita o acoplamento do tubo à conexão reta no fundo do tanque

\subsection{Realizaçáo dos ensaios}

Os ensaios foram realizados com a visualização da variação do nível de água do tanque durante o escoamento de água no sistema constituído pelo tanque e tubulaçáo nele acoplada. Nos primeiros ensaios realizados, verificou-se que o escoamento ocorria de forma muito rápida, dificultando a leitura dos níveis na fita métrica e a marcação simultânea dos tempos em um cronômetro. Essa dificuldade foi superada, de forma criativa, pelos alunos, que filmaram a superfície livre de líquido no tanque junto à fita métrica e a um celular, em cuja tela havia um cronômetro. Dessa forma, a partir de seleçâo das imagens capturadas no vídeo assim produzido, foi possível obter quadros com leituras de tempo e do nível do tanque. A Figura 7 ilustra como foi realizada essa filmagem.

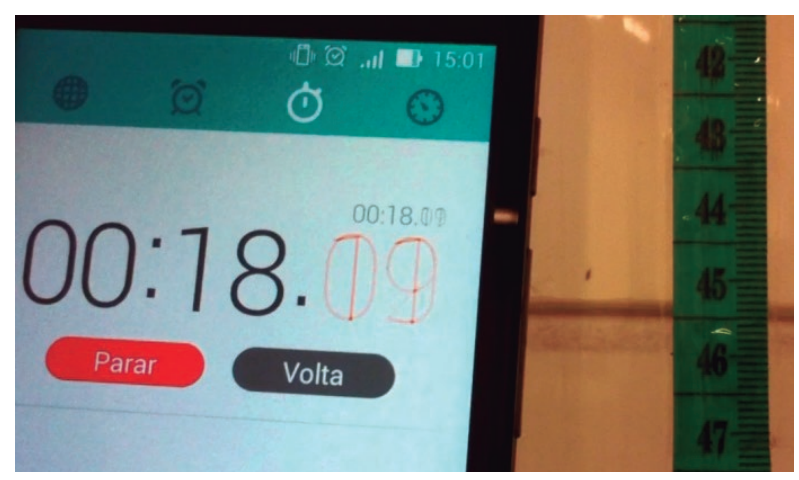

Figura 7 - Imagem ilustrativa da filmagem realizada.
Os dados assim obtidos foram inseridos numa tabela, associando o nível visualizado no tanque e o tempo decorrido desde o início do ensaio. A velocidade média de escoamento entre dois níveis consecutivos consiste no quociente entre a diferença de altura dos níveis e o tempo decorrido nesse escoamento entre eles.

Um corte longitudinal simplificado do equipamento utilizado é apresentado na Figura 8, e nela são indicados os diâmetros D1, D2 e D3, das seçóes transversais do escoamento no interior do tanque, no tubo metálico nele conectado e na conexão reta (peça 6), respectivamente.

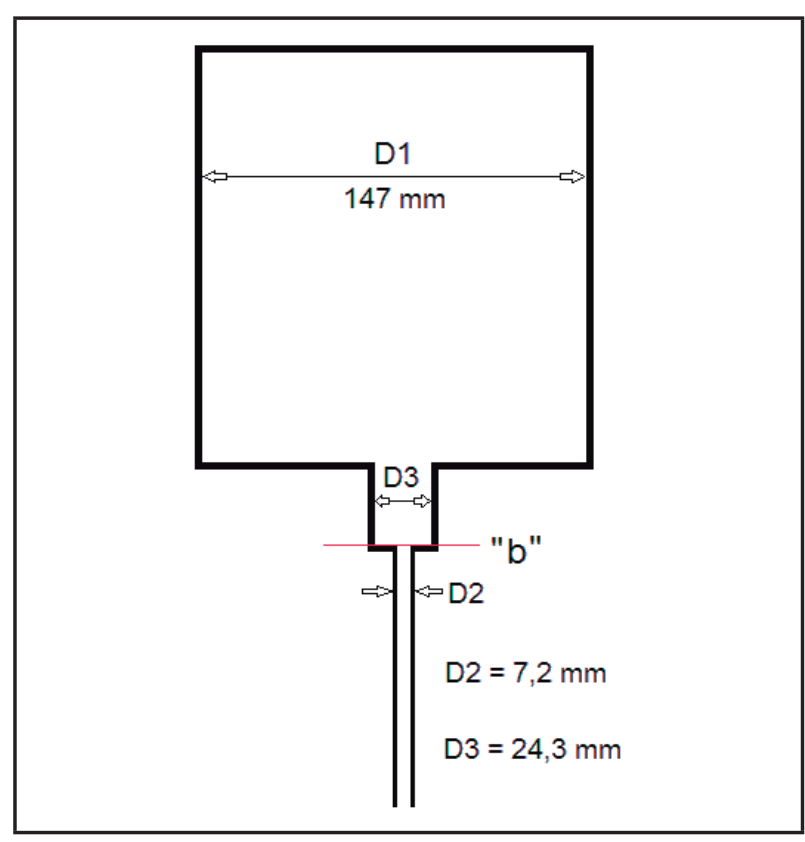

Figura 8 - Corte longitudinal simplificado do equipamento utilizado na realização dos ensaios.

O produto da área da seção transversal pela velocidade vertical do fluido numa seçâo fornece a vazão nessa seção. O princípio da conservação da massa, aplicado a um fluido incompressível (água), cujo escoamento possui seção transversal variável, conforme esquema apresentado na Figura 8, nos diz que:

$$
V_{1} \cdot A_{1}=V_{2} \cdot A_{2}=V_{3} \cdot A_{3}
$$

A velocidade $V_{1}$, calculada entre dois níveis consecutivos da tabela confeccionada, é atribuída ao ponto médio $\left(\boldsymbol{b}_{\boldsymbol{1}}\right)$ situado entre esses dois níveis. Os valores de $V_{2}$ e $V_{3}$ são calculados a partir da velocidade $V_{1}$.

A equaçáo (4), utilizada para o cálculo da perda de carga no sistema, é válida para um escoamento real e permanente; contudo, o escoamento analisado é transiente, pois a velocidade e o nível de líquido va- 
riam com o tempo. A hipótese de regime permanente pode ser, a princípio, empregada nos ensaios realizados, tendo em vista que os intervalos de cálculo das velocidades são muito curtos; e, consequentemente, não ocorre variaçôes significativas da velocidade nesses intervalos.

\subsection{Cálculo da perda de carga}

No intuito de avaliar a consistência dos dados obtidos nos ensaios, foram confrontados os valores experimentais de perda de carga no tubo com os valores teóricos correspondentes a essa perda de carga.

A perda de carga no sistema pode ser subdividida em três parcelas distintas, a saber:

a) no tanque e nos acessórios, com exceção do tubo nele acoplado;

b) na mudança súbita de área que ocorre na interface da conexão com a extremidade inicial do tubo;

c) no tubo propriamente dito.

A parcela correspondente ao item "a" constitui uma perda localizada, cujo valor de $\boldsymbol{K}$ pode ser calculado pela equação (6), num ensaio sem tubo acoplado. Nessa configuração, D3 é o diâmetro da seção de saída e, consequentemente, V3 é a velocidade a ser utilizada em (6).

O valor do coeficiente $\boldsymbol{K}_{\boldsymbol{b}}{ }^{1}$ para uma contração súbita é dado pela expressão:

$$
K_{b}=0,5 \cdot\left(1-\left(\frac{D_{s}}{D_{e}}\right)^{2}\right)
$$

Na equaçáo (9), $\boldsymbol{D}_{e}$ e $\boldsymbol{D}_{s}$ correspondem, respectivamente, aos diâmetros de entrada e saída da contraçáo. Dessa forma, a perda de carga presente na contração entre a conexão reta e o tubo nela acoplado, indicada por "b" na Figura 8, é fornecida pela expressão:

$$
h_{p}=0,5 \cdot\left(1-\left(\frac{D_{2}}{D_{3}}\right)^{2}\right) \cdot \frac{V_{2}^{2}}{2 \cdot g}
$$

A perda de carga no tubo corresponde à diferença entre a perda de carga total no sistema e o somatório das perdas em "a" e "b". Os ensaios foram realizados com um tubo cilíndrico, em aço comercial, de 7,2 $\mathrm{mm}$ de diâmetro e $300 \mathrm{~mm}$ de comprimento.

Essa foi a estratégia utilizada para o cálculo dos valores experimentais da perda de carga no tubo; sendo tais valores obtidos por meio das equaçóes (6), (9) e (10). Na Figura 9 é apresentado um fluxograma detalhando a metodologia de cálculo da perda de carga no tubo a partir dos dados experimentais.

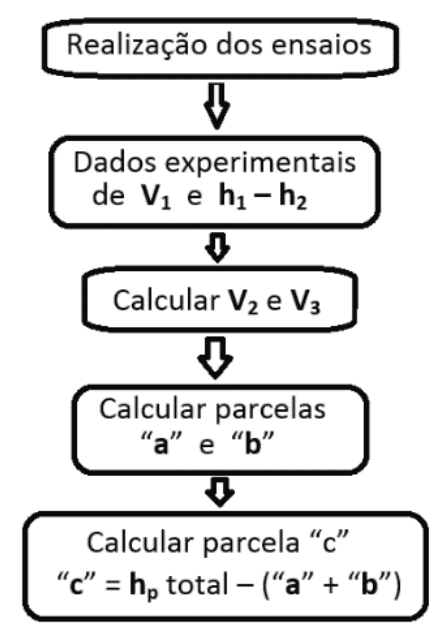

Figura 9 - Fluxograma ilustrativo do procedimento utilizado para cálculo da perda de carga experimental no tubo.

Na sequencia, é detalhado o procedimento utilizado para o cálculo da perda de carga teórica no tubo. Inicialmente, é necessário determinar a velocidade de escoamento $\left(\mathrm{V}_{2}\right)$ que produz termos de perda de carga cujas parcelas "a", "b" e "c" somadas com a carga cinética $\left(\mathrm{V}_{2}^{2} / 2\right.$.g $)$ na seçâo de saída se igualam à diferença de nível $\left(h_{1}-h_{2}\right)$ aplicada ao sistema, conforme indicado em (4). Essa velocidade teórica de escoamento é estimada para cada linha da tabela, e as três parcelas de perda de carga são calculadas com base nessa velocidade teórica; à exceção da parcela "a”, cujo cálculo emprega a velocidade na seção de diâmetro D3.

O fator de atrito $(\boldsymbol{f})$, necessário para o cálculo da perda de carga distribuída, é fornecido pela seguinte equação explícita (DAVIDSON et al., 1999):

$$
\frac{1}{\sqrt{f}}=-2 \cdot \log _{10}\left(\frac{\varepsilon}{3,7 \cdot D}\right)-\frac{5,16}{R e} \cdot \log _{10}\left(\frac{\varepsilon}{3,7 \cdot D}+\frac{5,09}{R e^{0,87}}\right)
$$

na qual $\varepsilon, \mathbf{D}$ e $\mathbf{R e}$ são a rugosidade média, o diâmetro interno e o valor do número de Reynolds para o escoamento no interior desse tubo. $\mathrm{O}$ tubo utilizado é de aço carbono comercial, cuja rugosidade é de 0,045 mm (FOX; McDONALD; PRITCHARD, 2004; BENRUTH; WILSON, 1989; KAMAND, 1988).

\footnotetext{
${ }^{1}$ Optou-se por utilizar o índice "b” para caracterizar que esse coeficiente está associado à perda de carga descrita no item "b”.
} 
Em síntese, o cálculo da velocidade teórica de escoamento é feito em um processo iterativo, no qual se arbitra um valor inicial de $V_{2}$, utilizado no cálculo do número de Reynolds, do fator de atrito e das parcelas que integram a perda da carga do sistema. A perda de carga total acrescida da carga cinética na seção de saída é comparada com a diferença de nível do sistema (membros direito e esquerdo, respectivamente, da equação (4)). Nas etapas subsequentes são selecionados sucessivos valores de $V_{2}$, até que se verifique a igualdade numérica entre os dois membros da equação (4). A Figura 10 detalha esse procedimento.

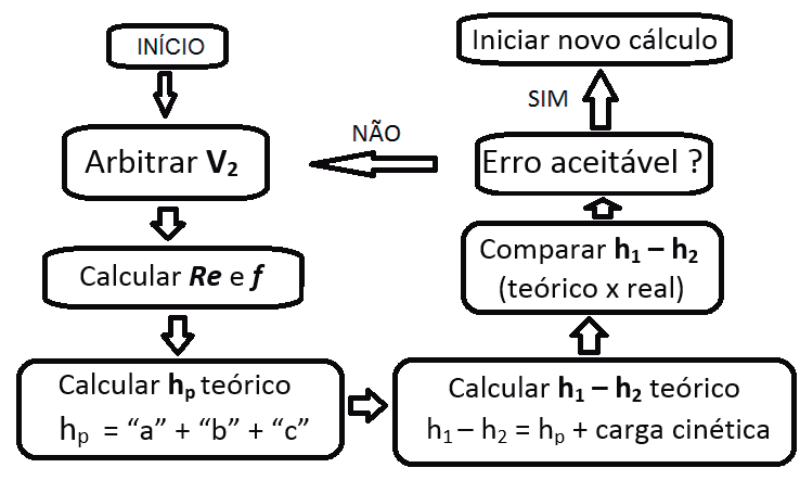

Figura 10 - Fluxograma ilustrativo do procedimento utilizado para cálculo da perda de carga teórica no tubo.

\section{RESULTADOS E DISCUSSĀO}

$\mathrm{Na}$ Tabela 1 sáo apresentados os dados experimentais obtidos, destacando os valores de velocidade e das três parcelas de perda de carga, bem como das diferenças de nível $\left(\mathrm{h}_{1}-\mathrm{h}_{2}\right)$ associadas aos valores de $\mathrm{V}_{1}$.

Tabela 1 - Valores experimentais das velocidades e das parcelas da perda de carga do sistema.

\begin{tabular}{c|c|c|c|c|c|c}
\hline $\begin{array}{c}\mathbf{h}_{1}-\mathbf{h}_{2} \\
(\mathbf{c m})\end{array}$ & $\begin{array}{c}\mathbf{V}_{1} \\
(\mathbf{c m} / \mathbf{s})\end{array}$ & $\begin{array}{c}\mathbf{V}_{2} \\
(\mathbf{c m} / \mathbf{s})\end{array}$ & $\begin{array}{c}\mathbf{V}_{\mathbf{3}} \\
(\mathbf{c m} / \mathbf{s})\end{array}$ & $\begin{array}{c}\mathbf{h} \text { “a” } \\
(\mathbf{c m})\end{array}$ & $\begin{array}{c}\mathbf{h} \text { "b" } \\
(\mathbf{c m})\end{array}$ & $\begin{array}{c}\mathbf{h} \\
\text { tubo } \\
(\mathbf{c m})\end{array}$ \\
\hline 123 & 0,718 & 299 & 26,3 & 2,40 & 19,9 & 54,9 \\
\hline 117 & 0,709 & 296 & 26,0 & 2,35 & 19,4 & 50,5 \\
\hline 111 & 0,688 & 287 & 25,2 & 2,21 & 18,3 & 48,4 \\
\hline 105 & 0,674 & 281 & 24,7 & 2,12 & 17,5 & 44,9 \\
\hline 99 & 0,637 & 266 & 23,3 & 1,89 & 15,7 & 45,4 \\
\hline 93 & 0,621 & 259 & 22,7 & 1,80 & 14,9 & 42,0 \\
\hline
\end{tabular}

$\mathrm{Na}$ Tabela 2 são apresentados os valores teóricos das velocidades e das parcelas de perda de carga sistema.
Tabela 2 - Valores teóricos das velocidades e das parcelas da perda de carga do sistema.

\begin{tabular}{c|c|c|c|c|c|c}
\hline $\begin{array}{c}\mathbf{h}_{1}-\mathbf{h}_{2} \\
(\mathbf{c m})\end{array}$ & $\begin{array}{c}\mathbf{V}_{1} \\
(\mathbf{c m} / \mathbf{s})\end{array}$ & $\begin{array}{c}\mathbf{V}_{2} \\
(\mathbf{c m} / \mathbf{s})\end{array}$ & $\begin{array}{c}\mathbf{V}_{3} \\
(\mathbf{c m} / \mathbf{s})\end{array}$ & $\begin{array}{c}\mathbf{h}_{\mathbf{p}} \text { “a” } \\
(\mathbf{c m})\end{array}$ & $\begin{array}{c}\mathbf{h}_{\mathbf{p}} \text { “b” } \\
(\mathbf{c m})\end{array}$ & $\begin{array}{c}\mathbf{h}_{\mathrm{p}} \\
\mathbf{t u b o} \\
(\mathbf{c m})\end{array}$ \\
\hline 123 & 0,698 & 291 & 25,5 & 2,27 & 18,8 & 58,6 \\
\hline 117 & 0,681 & 284 & 24,9 & 2,17 & 17,9 & 55,8 \\
\hline 111 & 0,662 & 276 & 24,2 & 2,05 & 16,9 & 52,7 \\
\hline 105 & 0,645 & 269 & 23,6 & 1,94 & 16,1 & 50,1 \\
\hline 99 & 0,626 & 261 & 22,9 & 1,83 & 15,1 & 47,1 \\
\hline 93 & 0,607 & 253 & 22,2 & 1,72 & 14,2 & 44,3 \\
\hline
\end{tabular}

A partir da análise das Tabelas 1 e 2 foi elaborado um gráfico que compara valores teóricos e experimentais da perda de carga no tubo (vide Figura 11). Os desvios foram inferiores a $11 \%$, sendo que o valor médio, calculado a partir dos módulos desses desvios, foi de $7,50 \%$.

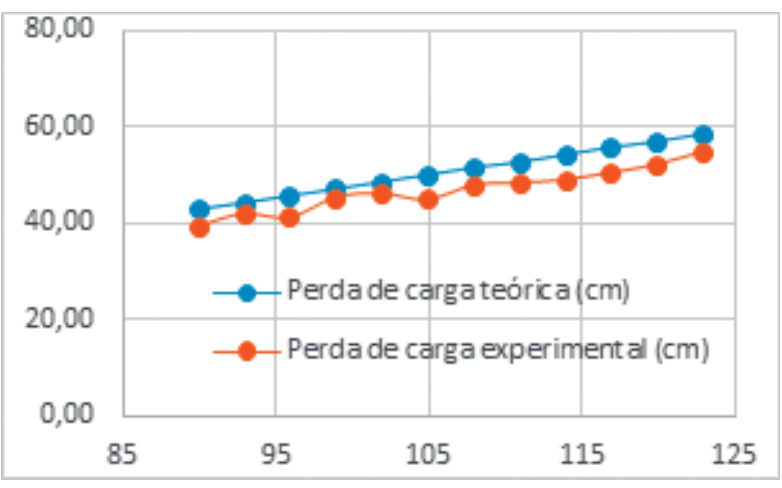

Figura 11 - Gráfico representativo da perda de carga no tubo em função da diferença de nível no sistema.

Considerando-se que a rugosidade do tubo, o fator de atrito e o valor tabelado de $\boldsymbol{K}$ para a contração súbita podem oscilar num percentual superior à diferença média registrada nos ensaios, é possível afirmar que os resultados obtidos foram satisfatórios. Dados experimentais obtidos em ensaios que avaliam a perda de carga em tubulaçóes apresentam incertezas que se situam entre 5 e 20\% (MELLO et. al., 1998; SAMPAIO et al., 2007).

As pequenas discrepâncias entre as velocidades teóricas e experimentais indicam que um balanço de energia em regime permanente pode ser aplicado ao sistema analisado. Essa simplificação, adotada no cálculo da perda de carga no sistema, mostrou-se válida para mediçôes de velocidade efetuadas durante uma pequena variação de nível no tanque, conforme detalhado na seção 3.2. Cabe ainda acrescentar que tais discrepâncias estão associadas a diversos fatores, entre 
os quais se inclui a precisão do equipamento empregado (cronômetro e vídeo de celular) na mediçáo do tempo e do nível de água no tanque; variações da seção transversal do tanque de acrílico ao longo de sua altura; e, a adoção de uma condiçãoo permanente para simular um regime transiente.

A necessidade de realização de uma série de cálculos para a elaboração gráficos e planilhas eletrônicas utilizadas na análise dos dados experimentais exigiu dos alunos a revisão de conceitos teóricos e a manipulação de equaçóes, de modo que o experimento proposto constitui uma excelente ferramenta didática (FEISEL; PETERSON, 2002).

O baixo custo de construção do tanque hidráulico apresentado nesse artigo torna o experimento acessível a instituições de ensino com limitações orçamentárias e dificuldades na obtenção de recursos financeiros para aquisição de equipamentos de laboratório.

O experimento proposto não envolve o consumo de reagentes, tampouco a necessidade de manutençâo ou de assistência técnica especializada; evitando complicaçôes que, muitas vezes, resultam na inoperância de equipamentos mais sofisticados e cujo custo de aquisição é muito elevado.

A simplicidade e robustez da estrutura do tanque, bem como a facilidade de realização dos ensaios, permitem que o aluno trabalhe de forma mais autônoma, sem uma supervisão excessiva por parte do professor (KRIVICKAS, 2007; FEISEL; ROSA, 2005). Tal fato foi constatado nos primeiros ensaios realizados, quando os alunos discutiram a melhor maneira de efetuar a leitura e a marcação dos intervalos de tempos e propuseram uma solução criativa e eficiente. A vinculação do processo ensino-aprendizagem ao experimento proposto resultou, além do empenho e dedicação constatados na elaboração dessa solução, num melhor desempenho nas avaliações escritas e na produção dos relatórios (ALVES FILHO, 2000; SOARES, 1997; SAAD, 1977; ARAÚJO; ABIB, 2003).

\section{CONCLUSÓES}

A execução do experimento requer a realização de uma série de cálculos e a inserção das fórmulas algébricas numa planilha eletrônica. Tais atividades são precedidas de um trabalho em equipe para realizar as filmagens, propiciando uma mobilizaçáo dos alunos no sentido de executar as tarefas propostas. A robustez do equipamento e simplicidade dos ensaios possibilitam que o aluno trabalhe de forma mais autônoma, fato esse que reforça a cooperação entre os alunos.

O baixo custo de construção do tanque hidráulico e as características do experimento proposto viabilizam sua utilização em instituiçóes de ensino com dificuldades para aquisição de equipamentos e que necessitam ou desejam incluir aulas experimentais de Mecânica dos Fluidos em seus cursos de graduação.

Os resultados experimentais obtidos foram satisfatórios, e os desvios constatados entre valores teóricos e experimentais são similares àqueles relatados em outros ensaios que avaliam a perda de carga em tubulações.

Uma alternativa para minimizar as discrepâncias entre valores teóricos e experimentais da velocidade é a manutençâo de um nível constante de água no tanque, caracterizando um regime permanente de fato. Nesse caso, mediçôes do volume escoado no tempo permitem calcular as velocidades; sendo que maiores intervalos de tempo resultam em valores mais precisos de velocidade e, consequentemente, menores desvios entre valores teóricos e experimentais de perda de carga no tubo reto. As modificações necessárias no equipamento, bem como alteraçôes nas formulações matemáticas adotadas para simular o sistema modificado, nos levam a concluir que essa alternativa deve ser implementada e avaliada em um trabalho futuro.

Ensaios de perda de carga para tubos de diferentes comprimentos e diâmetros, facilitados pelo emprego de tubulações intercambiáveis nesse tanque hidráulico, fornecerão uma grande quantidade de dados experimentais. A realização desses ensaios enseja trabalhos futuros, voltados para uma análise mais ampla dos fatores que afetam a perda de carga em tubulações.

\section{REFERÊNCIAS}

ALVES FILHO, J. P. Regras da transposição didática aplicada ao laboratório didático. Caderno Catarinense de Ensino de Física, v. 17, n.2, p.174-188, ago. 2000.

ARAÚJO, M. S.; ABIB, M. L. S. Atividades experimentais no ensino de física: diferentes enfoques, diferentes finalidades. Revista Brasileira de Ensino de Física, São Paulo, v.25, n.2, p.176-194, 2003.

BERNUTH, R.D. von; WILSON, T. Friction factors for small diameter plastic pipes. Journal of Hydraulics Engineering, New York, v.115, n.2, p.183-192, 1989.

D’ANGELO, J. V. H.; ZEMP, R. J. Experimentos em sala de aula com forma de estimula a aprendizagem de concei- 
tos fundamentais em engenharia. Revista Ensino Superior, v. 5, n. 13, p. 6-17, abr./jun. 2014.

DAVIDSON, J.W. et al. Approximators for the Colebrook-White formula obtained through a hybrid regression method. Exeter: Universidade de Exeter, 1999.

FEISEL, L.D.; PETERSON, G.P. The challenge of the laboratory in engineering education. Journal of Engineering. Education, v.91, n.4, p.367-368, 2002.

FEISEL, L.D.; ROSA, A.J. The role of the laboratory in undergraduate engineering education. Journal of Engineering. Education, v.94, n.1, p.121-130, 2005.

FOX, R.W.; McDONALD, A.T.; PRITCHARD, P.J. Introduçáo à Mecânica dos Fluidos. 6. Ed. São Paulo: LTC, 2004 .

KAMAND, F. Z. Hydraulic friction factors for pipe flow. Journal of Irrigation and Drainage Engineering, New York, v. 114. n.2. p.311-323, maio 1988.

KRIVICKAS, R. V.; KRIVICKAS, J. Laboratory Instruction in Engineering Education. Global Journal of Engineering Education, v.11, n.2, p.191-196, 2007.

LUDKE, E. et al. Um experimento para ensino de conceitos de transferência de calor em laboratório de física. Revista Brasileira de Ensino de Física, São Paulo, v.35, n.1, p. 2-4, jan./mar. 2013

MELLO, C. R. de; FERREIRA, D. F.; CARVALHO, J. A. Equaçôes explícitas para o fator de atrito da equação de Darcy-Weisbach. In: CONGRESSO BRASILEIRO DE ENGENHARIA AGRÍCOLA, 27., 1998, Poços de Caldas. Anais ... Poços de Caldas: Sociedade Brasileira de Engenharia Agrícola, 1998. v.1, p. 142-144.

SAAD, F. D. O laboratório didático de física no ensino de física. Tese (doutorado) - Instituto de Física da USP, São Paulo, 1983.

SAMPAIO, S. C. et al. Equação de Hazen-Williams corrigida para água residuária proveniente da suinocultura. Revista Brasileira de Engenharia Agrícola e Ambiental, v. 11, n. 1 , p. 5-10, 2007.

SHAMES, I. H. Mecânica dos Fluidos: princípios básicos. 2. Ed. São Paulo: Edgard Blucher, 1996. v. 1.

SCHLICHTING, H. Boundary Layer Theory. $8^{\text {th }}$ Ed. [S.1.]: Springer Verlag,2001.

SOARES, V.L. L. Laboratório didático de Física no ciclo básico da universidade. Dissertação (Mestrado) - Instituto de Física da USP, São Paulo, 1977.

WHITE, F.M. Viscous Flow. $3^{\text {rd }}$ ed. [S.1.]: McGraw Hill, 2006 . 